Check for updates

Cite this: RSC Adv., 2018, 8, 32899

\title{
Self-curing triphenol A-based phthalonitrile resin precursor acts as a flexibilizer and curing agent for phthalonitrile resin $\uparrow$
}

\author{
Yue Hu, Zhihuan Weng, (D)* Yu Qi, Jinyan Wang, (D) Shouhai Zhang, Cheng Liu, \\ Lishuai Zong and Xigao Jian (iD
}

Major problems currently limiting the widespread application of phthalonitrile resins are the high precursor melting point and volatility of the curing agent. Herein, a novel self-curing triphenol A-based phthalonitrile resin precursor (TPPA-Ph) was successfully synthesized by reacting $\alpha, \alpha, \alpha^{\prime}$-tris(4-hydroxyphenyl)-1-ethyl-4isopropylbenzene (TPPA) with 4-nitrophthalonitrile (NPh) via nucleophilic substitution. The presence of residual phenolic hydroxyl groups in the TPPA-Ph precursor promoted the curing reaction of phthalonitrile resin in the absence of an additional curing reagent. Self-cured TPPA-Ph resins exhibited relatively low melting points (less than $100{ }^{\circ} \mathrm{C}$ ), high thermal stability, and a wide processing window (116 ${ }^{\circ} \mathrm{C}$ ). Furthermore, the TPPA-Ph precursors contained phenolic hydroxyl and cyano groups that can be used as flexibilizers and curing agents to optimize other phthalonitrile resins. Resorcinol-based phthalonitrile resin (DPPH) cured with various amounts of TPPA-Ph possessed excellent thermal and thermo-oxidative stability with a $5 \%$ weight loss temperature exceeding $530{ }^{\circ} \mathrm{C}, \mathrm{T}_{\mathrm{g}} \mathrm{s}$ above $380{ }^{\circ} \mathrm{C}$, and a wide processing window and time. Therefore, as a novel precursor and curing agent for phthalonitrile resins, the triphenol A-based phthalonitrile resin is an ideal resin matrix for high-performance composites with broad application prospects in aerospace, shipping, machinery, and other high-tech fields.

Received 18th August 2018

Accepted 11th September 2018

DOI: $10.1039 / c 8 r a 06926 c$

rsc.li/rsc-advances simple bisphenol-based phthalonitriles ${ }^{10}$ have been designed and synthesized. However, the curing rate of the phthalonitrile monomer is very slow, taking nearly one hundred hours to produce a significant gel at $280{ }^{\circ} \mathrm{C}$. Subsequently, Keller and coworkers found that mixing phthalonitrile monomers and aromatic amines effectively promoted the curing of phthalonitrile monomers, with the curing rate related to the alkalinity of the aromatic amines. ${ }^{11}$ Curing additives that can catalyze the production of high-performance phthalonitrile resin include strong organic acids,${ }^{12}$ organic amines, ${ }^{13}$ organic acid/amine salts, ${ }^{14}$ metals, ${ }^{15}$ and metal salts. ${ }^{16}$ Currently, aromatic diamines, such as 1,3-bis(3-aminophenoxy)benzene ( $m$-APB), diamino diphenyl sulfone (DDS), and 1,3-bis(3,4-dicyanophenoxy)benzene (BDB), are commonly used as curing agents for phthalonitrile resins. Although the amount of aromatic diamine curing agent added can adjust the rate of phthalonitrile curing, the aromatic amines ${ }^{17}$ are easily decomposed. Adding too much curing agent produces voids in the material during high-temperature treatment, which affects the mechanical properties of the material. To resolve this problem, a stable curing agent is needed to prevent volatilization during high-temperature curing and, therefore, improve the mechanical performance of phthalonitrile resin.

Meanwhile, another problem of phthalonitrile resin is the high melting point of the phthalonitrile monomer, which is caused by its larger molecular rigidity (usually greater than $200{ }^{\circ} \mathrm{C}$ ). ${ }^{18-21}$ For
State Key Laboratory of Fine Chemicals, Liaoning High Performance Resin Engineering Research Center, Department of Polymer Science \& Materials, Dalian University of Technology, Dalian 116024, China.E-mail: zweng@dlut.edu.cn

$\dagger$ Electronic supplementary information (ESI) available. See DOI: $10.1039 / \mathrm{c} 8 \mathrm{ra06926c}$ 
example, bisphenol A-based phthalonitrile has a melting point of $195{ }^{\circ} \mathrm{C}$, while the melting point of biphenyldiol-based phthalonitrile is $235{ }^{\circ} \mathrm{C} .{ }^{17}$ However, phthalonitrile resin curing can be conducted after the phthalonitrile monomer has been melted. Therefore, the processing temperature window from the melting point to the gel point is too narrow, making it difficult to process and mold the phthalonitrile resin. Therefore, the synthesis of a phthalonitrile monomer with a low melting point is key to the development of phthalonitrile resin.

In brief, the major problems currently limiting the widespread application and development of phthalonitrile resin are the high precursor melting point, curing agent volatility, high curing temperatures, and long curing times. ${ }^{22,23}$ Therefore, research into new resin precursors and novel curing agents can effectively solve these issues. ${ }^{24-26}$ In our laboratory, we have demonstrated introducing phthalazinone groups into phthalonitrile resins enhances their thermal stability. ${ }^{27}$ Meanwhile, the new curing agent has been screened to improve the phthalonitrile resin processing properties. ${ }^{28,29}$

Herein, a new type of multifunctional phthalonitrile resin (TPPA-Ph) containing a triphenol A structure was prepared using $\alpha, \alpha, \alpha^{\prime}$-tris(4-hydroxyphenyl)-1-ethyl-4-isopropylbenzene (TPPA) as a feedstock in a one-pot reaction. Using TPPA as a monomer was advantageous for the construction of phthalonitrile resins. Firstly, TPPA-based phthalonitrile precursor TPPA-Ph has a relatively low melting point due to the TPPA monomer containing an alkyl flexible center and isopropyl benzene flexible group. Secondly, by regulating the reaction point, the phthalonitrile resin system retains a certain amount of phenolic hydroxyl groups after the reaction, which can activate phthalonitrile resin self-curing without an additional curing agent. Therefore, the formation of voids due to the addition of external curing agent is avoided, resulting in an excellent phthalonitrile resin. Thirdly, phenolic hydroxyl groups in the precursor can also be used as a flexibilizer to optimize other phthalonitrile resins, which improves the processing performance of phthalonitrile resin. The cure kinetics, and thermal and dynamic mechanical properties, were also investigated.

\section{Experimental}

\section{Materials}

All materials were obtained from commercial suppliers and used without further purification. $\alpha, \alpha, \alpha^{\prime}$-Tris(4-hydroxyphenyl)-1-ethyl4-isopropylbenzene (TPPA) was supplied by Tokyo Chemical Industry Development Co., Ltd. 4-Nitrophthalonitrile (NPh) was purchased from Wuhan Yinguan Chemical Co., Ltd. Anhydrous potassium carbonate $\left(\mathrm{K}_{2} \mathrm{CO}_{3}\right)$ was obtained from Tianjin Damao Chemical Reagent Factory, ground into powder in a mortar, and dried in vacuum prior to use. Resorcinol was purchased from Sinopharm Chemical Reagent Co., Ltd. Dimethyl sulfoxide (DMSO) was supplied by Tianjin Fuyu Fine Chemical Co., Ltd.

\section{Synthesis of self-curing TPPA-based phthalonitrile resin precursor (TPPA-Ph)}

TPPA-Ph was synthesized by regulating the reaction conditions to control the phenolic hydroxyl group content in the system.
Typically, TPPA (10 g), NPh (4.28 g), pulverized anhydrous $\mathrm{K}_{2} \mathrm{CO}_{3}(4.88 \mathrm{~g})$, and DMSO $(100 \mathrm{~mL})$ were added into a $250 \mathrm{~mL}$ round-bottom flask with magnetic stirring. The resulting solution was heated at $40{ }^{\circ} \mathrm{C}$ for $24 \mathrm{~h}$ under a nitrogen atmosphere. When the reaction ended, the product was poured into deionized water $(1000 \mathrm{~mL})$ and adjusted to neutral $\mathrm{pH}$ with dilute $\mathrm{HCl}$ solution. The resultant off-white solid was washed with deionized water until the filtrate was neutral and dried at $70{ }^{\circ} \mathrm{C}$ for $24 \mathrm{~h}$ in a vacuum oven. Similar to this process, different TPPA$\mathrm{Ph}$ precursor systems were synthesized by changing the reaction conditions, such as reactant proportion and reaction temperature.

\section{Synthesis of resorcinol-based phthalonitrile resin precursor (DPPH)}

Resorcinol (11 g), NPh (35.46 g), pulverized anhydrous $\mathrm{K}_{2} \mathrm{CO}_{3}$ $(20.71 \mathrm{~g})$, and DMSO $(110 \mathrm{~mL})$ were added into a $250 \mathrm{~mL}$ threenecked flask. The resulting solution was stirred at $30{ }^{\circ} \mathrm{C}$ for $48 \mathrm{~h}$ under a nitrogen atmosphere. The product mixture was then poured into hot deionized water and adjusted to neutral $\mathrm{pH}$ with dilute $\mathrm{HCl}$ solution. The wet filter cake was washed with deionized water and ethanol until the filtrate was neutral, and dried at $50{ }^{\circ} \mathrm{C}$ for $24 \mathrm{~h}$ in a vacuum oven.

\section{Thermal curing of phthalonitrile resins}

TPPA-Ph was stirred at $100{ }^{\circ} \mathrm{C}$ in a reaction vessel until melting and pouring into a mold for thermal curing. The curing process was performed at $200{ }^{\circ} \mathrm{C}$ for $2 \mathrm{~h}, 250{ }^{\circ} \mathrm{C}$ for $2 \mathrm{~h}, 350{ }^{\circ} \mathrm{C}$ for $2 \mathrm{~h}$, and $400{ }^{\circ} \mathrm{C}$ for $2 \mathrm{~h}$ in a muffle furnace.

DPPH and TPPA-Ph (mass ratios of 10\%, 20\%, 25\%, and $30 \%$ ) were ground evenly and stirred in a reaction vessel at $180^{\circ} \mathrm{C}$ until melting. The mixtures were then cured at $200{ }^{\circ} \mathrm{C}$ for $2 \mathrm{~h}, 250{ }^{\circ} \mathrm{C}$ for $2 \mathrm{~h}, 300{ }^{\circ} \mathrm{C}$ for $2 \mathrm{~h}, 350{ }^{\circ} \mathrm{C}$ for $2 \mathrm{~h}, 375^{\circ} \mathrm{C}$ for $2 \mathrm{~h}$, and $400{ }^{\circ} \mathrm{C}$ for $2 \mathrm{~h}$ in a muffle furnace. As a reference, $5 \%$ (mass ratio) DDS was mixed with DPPH and cured under identical conditions.

\section{Characterizations}

${ }^{1} \mathrm{H}$ NMR spectra were obtained using a Bruker Avance II $400 \mathrm{M}$ nuclear magnetic resonance (NMR) spectrometer, using deuterated dimethylsulfoxide (DMSO- $\mathrm{d}_{6}$ ) as solvent and operating at $25{ }^{\circ} \mathrm{C}$. Fourier transform infrared (FTIR) spectra were collected on a Thermo Nicolet Nexus 470 FTIR spectrometer using an attenuated total reflectance (ATR) accessory in the range $4000-650 \mathrm{~cm}^{-1}$ at room temperature. High-performance liquid chromatography (HPLC) was performed with a HP-1100 liquid chromatography system. Differential scanning calorimetry (DSC) was performed with a Mettler Toledo DSC1 calorimeter under nitrogen flow $\left(50 \mathrm{~mL} \mathrm{~min}^{-1}\right)$ at different heating rates $\left(5,10,15\right.$, and $\left.20{ }^{\circ} \mathrm{C} \mathrm{min}^{-1}\right)$ over the temperature range 25-350 ${ }^{\circ} \mathrm{C}$. DSC scans showed the curing temperature for maximum peaks $\left(T_{\mathrm{p}}\right)$ and the curing kinetics of TPPA-Ph with different compositions. Thermogravimetric analysis (TGA) curves were obtained using a Mettler TGA1 Instrument at a heating rate of $10{ }^{\circ} \mathrm{C} \mathrm{min}^{-1}$ under a nitrogen or air purge (50 $\mathrm{mL} \min ^{-1}$ ) from 30 to $800{ }^{\circ} \mathrm{C}$. Rheological behavior was 
investigated using a TA Instrument AR-2000 rheometer. Measurements were made at a frequency of $1 \mathrm{~Hz}$ under a strain of $0.02 \mathrm{~N}$ and employed at a heating rate of $3{ }^{\circ} \mathrm{C} \mathrm{min}^{-1}$ from room temperature to $400{ }^{\circ} \mathrm{C}$. Samples for rheometric testing were compressed into a cylinder (diameter, $25 \mathrm{~mm}$; thickness, 1 $\mathrm{mm}$ ). Dynamic mechanical analysis (DMA) was performed using a TA Instruments Q800 dynamic mechanical analyzer at a frequency of $1 \mathrm{~Hz}$ from room temperature to $380{ }^{\circ} \mathrm{C}$ with a heating rate of $3{ }^{\circ} \mathrm{C} \min ^{-1}$.

\section{Curing kinetic analysis}

The kinetic analysis of thermal transformations is based on the assumption that the transformation rate $(\mathrm{d} \alpha / \mathrm{d} t)$ during a reaction is the product of two functions, one depending completely on the temperature $(T)$, and the other depending completely on the fraction transformed $(\alpha):^{30}$

$$
\frac{\mathrm{d} \alpha}{\mathrm{d} t}=K(T) f(\alpha)
$$

where $K(T)$ is a temperature-dependent reaction rate constant, and $f(\alpha)$ is a differential conversion function dependent on the curing mechanism. ${ }^{31}$

The temperature-dependent function can be easily determined using the Arrhenius equation: ${ }^{30}$

$$
K(T)=A \exp \left(-\frac{E_{\alpha}}{R T}\right)
$$

where $A$ is the pre-exponential factor, $E_{\alpha}$ is the apparent activation energy, $R$ is the universal gas constant, and $T$ is the absolute temperature.

The frequently applied methods of determining $E_{\alpha}$ are the Kissinger, Ozawa, and Friedman methods for non-isothermal reactions. Using the Starink method is recommended due to higher accuracy of $E_{\alpha}$ according to the ICTAC kinetic project. ${ }^{32}$

$$
\ln \frac{\beta_{i}}{T_{\alpha, i}{ }^{1.92}}=\text { Const }-1.0008\left(\frac{E_{\alpha}}{R T_{\alpha}}\right)
$$

where $T_{\alpha, i}$ is the temperature at which the extent of conversion $\alpha$ is reached under the $i$ th heating rate, and $\beta$ is the heating rate.

\section{Results and discussion}

\section{Preparation and structure of TPPA-Ph precursors}

As shown in Scheme 1, the TPPA monomer consists of an alkyl flexible center, isopropyl benzene flexible group, and three phenolic hydroxyl groups with different reactivities. In a polar aprotic solvent, the three phenolic hydroxyl groups of TPPA were reacted with NPh under using an inorganic base as catalyst to afford the multifunctional TPPA-based phthalonitrile resin precursors. By controlling the reaction conditions, such as reactant proportion and reaction temperature, we obtained TPPA-Ph precursors with different phenolic hydroxyl contents, as shown in Table 1. Comparing the ${ }^{1} \mathrm{H}$ NMR spectra of the raw material and the TPPA-Ph precursors indicated that the presence of certain residual phenolic hydroxyl groups $(\delta=9.12-9.34$ ppm) was key to the self-curing nature of TPPA-based phthalonitrile resin precursor systems (Fig. 1). ${ }^{33,34}$ However, characterizing each proton peak was difficult due to the resultant products being mixtures, as shown by ${ }^{1} \mathrm{H}$ NMR analysis. In the FT-IR (ATR) spectra, absorption bands at around $3400 \mathrm{~cm}^{-1}$, $2970 \mathrm{~cm}^{-1}, 2229 \mathrm{~cm}^{-1}$, and $1012 \mathrm{~cm}^{-1}$ were attributed to the stretching vibrations of phenolic hydroxyl groups, carbonhydrogen bonds, cyano groups, and aromatic ether groups, as shown in Fig. 2. Notably, the peak at $3400 \mathrm{~cm}^{-1}$ was very weak in the case of TPPA-Ph (P1) due to the low content of phenolic hydroxyl groups. Both the ${ }^{1} \mathrm{H}$ NMR spectra and FT-IR (ATR) spectra demonstrated the successful synthesis of self-curing TPPA-based phthalonitrile resin precursors.

The presence of residual phenolic hydroxyl groups in TPPA$\mathrm{Ph}$ promoted curing of the phthalonitrile resin. The phenolic hydroxyl content, structure, and distribution of the TPPA-Ph precursors were determined using HPLC and HPLC-MS (Fig. 1S $\dagger$ ). These results indicated that five compounds were present in total, whose structures were classified as TPPA-PhN1-3, and a total of three types according to the different phenolic hydroxyl contents. There were two mono-substituted compounds (TPPA-Ph-N1-A and TPPA-Ph-N1-B), two bissubstituted compounds (TPPA-Ph-N2-A and TPPA-Ph-N2-B) and one tri-substituted compound (TPPA-Ph-N3) in the TPPA$\mathrm{Ph}$ precursor systems. Different distributions of the TPPA-Ph precursor systems were synthesized by changing the reaction parameters, such as reactant proportion and reaction temperature (Table 1). Increasing the molar ratio of NPh to TPPA and the reaction temperature led to a decrease in the proportion of mono-substituted compounds (TPPA-Ph-1; from $44.0 \%$ to $0.0 \%$ ), while the proportion of the tri-substituted compound (TPPA-Ph-3) was increased from $12.9 \%$ to $90.2 \%$. Using a TPPA : $\mathrm{K}_{2} \mathrm{CO}_{3}$ : NPh molar ratio of $1: 1.5: 1.05$ and a temperature of $25{ }^{\circ} \mathrm{C}$, the TPPA-Ph precursor system contained $44.0 \%$ of the mono-substituted compounds (TPPA-Ph-N1) and 43.1\% of the bis-substituted compounds (TPPA-Ph-N2), which afforded the majority of phenolic hydroxyl groups that act as curing centers to facilitate TPPA-based phthalonitrile resin curing.

\section{Performance of self-curing TPPA-based phthalonitrile resin}

Non-isothermal DSC analysis was used to investigate the curing kinetics of TPPA-Ph precursors at heating rates of 5, 10, 15, and $20 \mathrm{~K} \mathrm{~min}^{-1}$ under a nitrogen atmosphere. As shown in Fig. 3, TPPA-Ph (P4) melted at $97.2{ }^{\circ} \mathrm{C}$ and the exothermic peak at $248.9{ }^{\circ} \mathrm{C}$ confirmed that TPPA-Ph curing had proceeded successfully without an additional curing reagent. According to basic kinetic theory, ${ }^{35,36}$ the shape of the curing kinetic curves can be used to determine the reaction path. Graphs that plot conversion a as a function of curing temperature (Fig. 2S $\dagger$ ) using data obtained from dynamic DSC (Fig. 3) show typical sigmoid shapes that are characteristic of curing reactions that follow an autocatalytic mechanism. ${ }^{37}$ The Starink method is normally recommended for conducting isoconversional analysis of dynamic DSC data. Therefore, $\ln \left(\beta_{\mathrm{i}} / T_{\alpha, i}{ }^{1.92}\right)$ vs. $1 / T_{\alpha}$ values for the same fractional extent of conversion for a series of dynamic DSC experiments conducted at various heating rates can be plotted to afford a straight line with a gradient of $-1.0008 E_{\alpha} / R$. Plots were obtained for TPPA-Ph (P4) for 

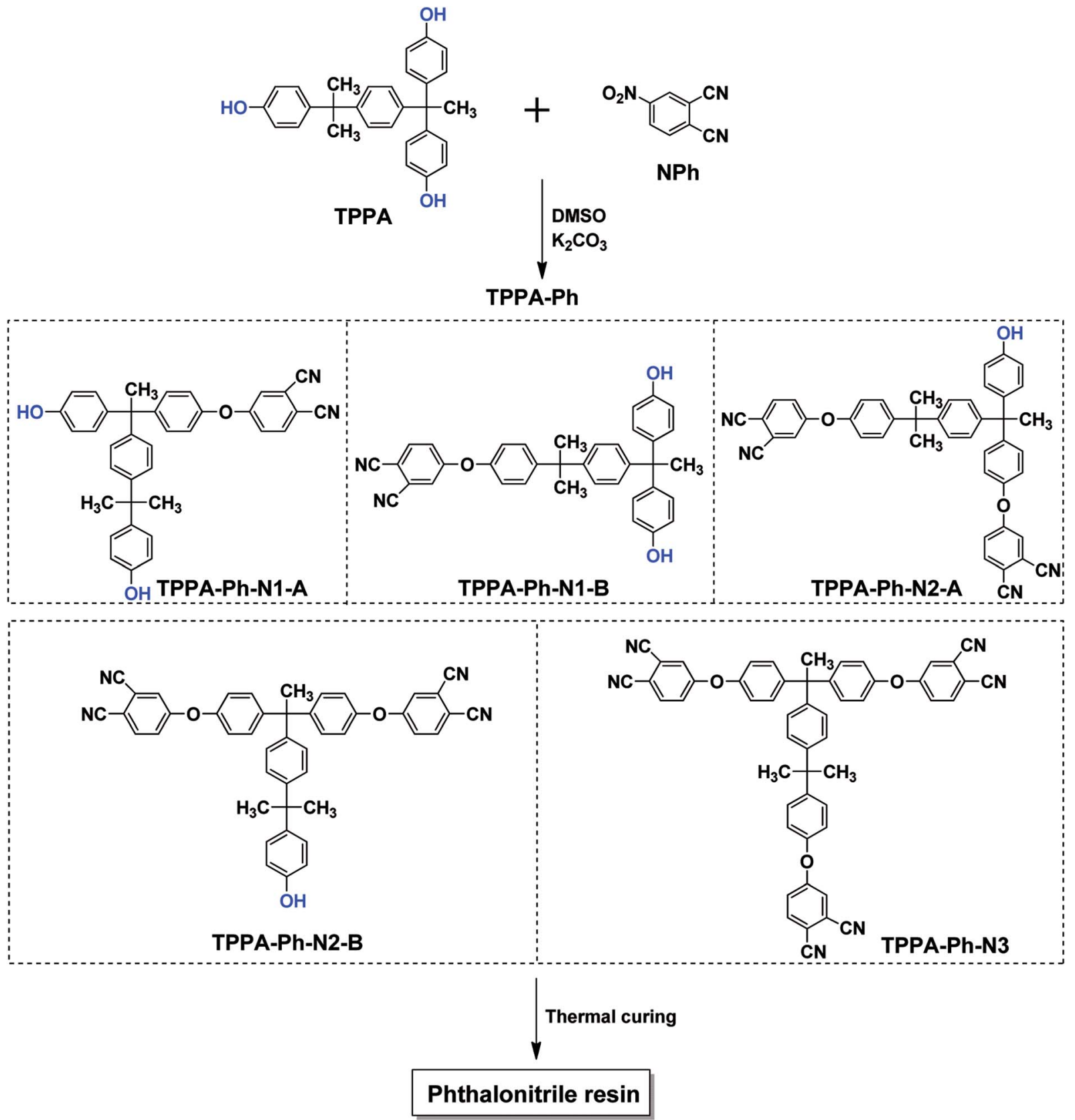

Scheme 1 Synthesis of multifunctional TPPA-based phthalonitrile resin precursor (TPPA-Ph).

Table 1 Component contents in TPPA-Ph products

\begin{tabular}{lccl}
\hline TPPA-Ph & TPPA-Ph-N1 (\%) & TPPA-Ph-N2 (\%) & TPPA-Ph-N3 (\%) \\
\hline P1 & 0.0 & 9.8 & 90.2 \\
P2 & 11.6 & 42.3 & 46.1 \\
P3 & 28.0 & 29.5 & 42.5 \\
P4 & 44.0 & 43.1 & 12.9
\end{tabular}

a conversion range of $0.1-0.9$, with a good linear relationship observed in both cases, as shown in Fig. 3S. $\dagger$ Repeating the procedure, $E_{\alpha}$ values corresponding to various a values can then be obtained from the DSC curing curves, with the change in apparent activation energy with respect to the degree of conversion shown in Fig. 4S. $\dagger$ The average value of $E_{\alpha}$ was $127.7 \mathrm{~kJ} \mathrm{~mol}^{-1}$ for the TPPA-Ph (P4) precursor, which was similar to those reported previously for this type of resin. ${ }^{38}$ Notably, TPPA-Ph (P4) showed a gradual decrease in activation energy at conversion levels greater than 0.5 in Fig. $4 \mathrm{~S}, \dagger$ which can be attributed to changes in the rate-determining step of a diffusion-controlled process. ${ }^{39}$

The self-curing reactions of TPPA-Ph precursors were conducted under temperature-programmed conditions $\left(200{ }^{\circ} \mathrm{C}\right.$ for $2 \mathrm{~h}, 250{ }^{\circ} \mathrm{C}$ for $2 \mathrm{~h}, 350{ }^{\circ} \mathrm{C}$ for $2 \mathrm{~h}$, and $400{ }^{\circ} \mathrm{C}$ for $2 \mathrm{~h}$ ). The thermal stabilities of the resulting TPPA-Ph resins were 


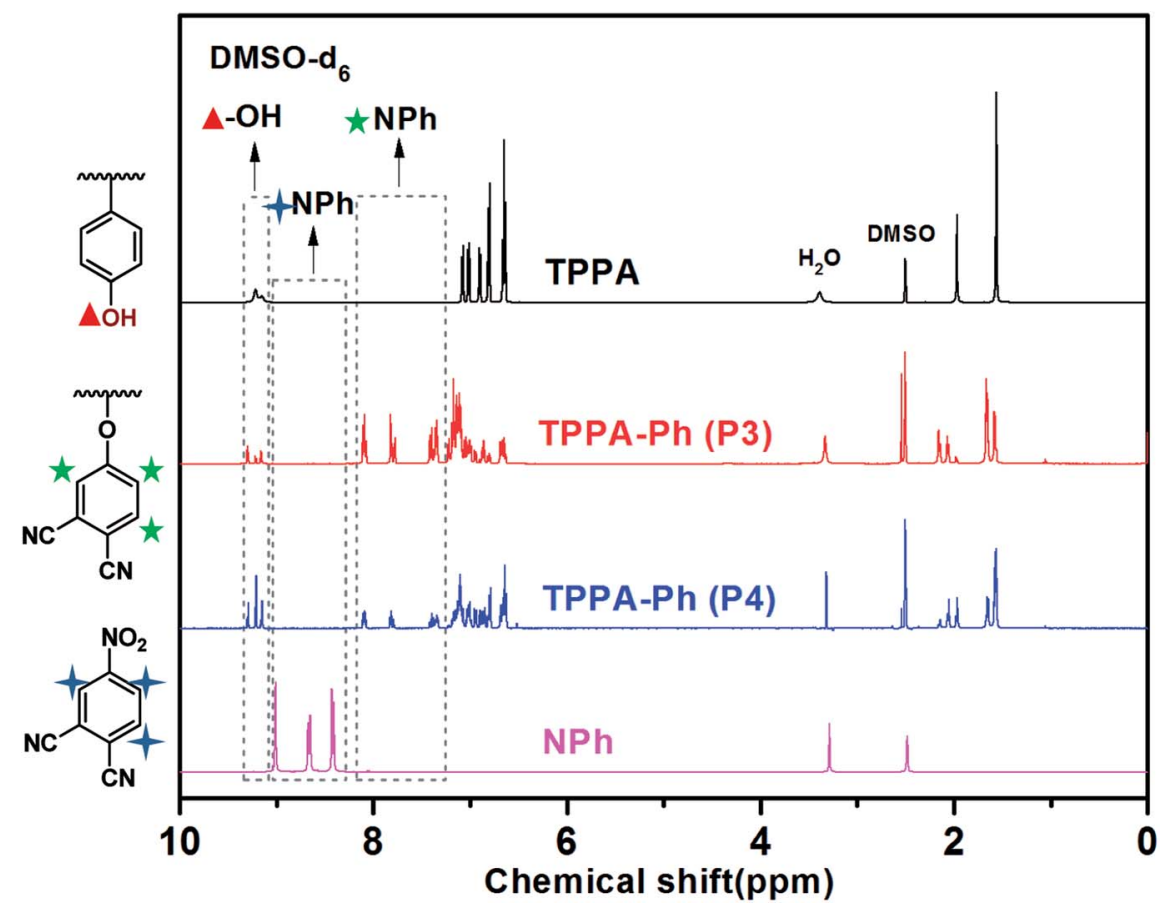

Fig. $1{ }^{1} \mathrm{H}$ NMR spectra of TPPA, NPh, and TPPA-Ph precursors with different compositions.

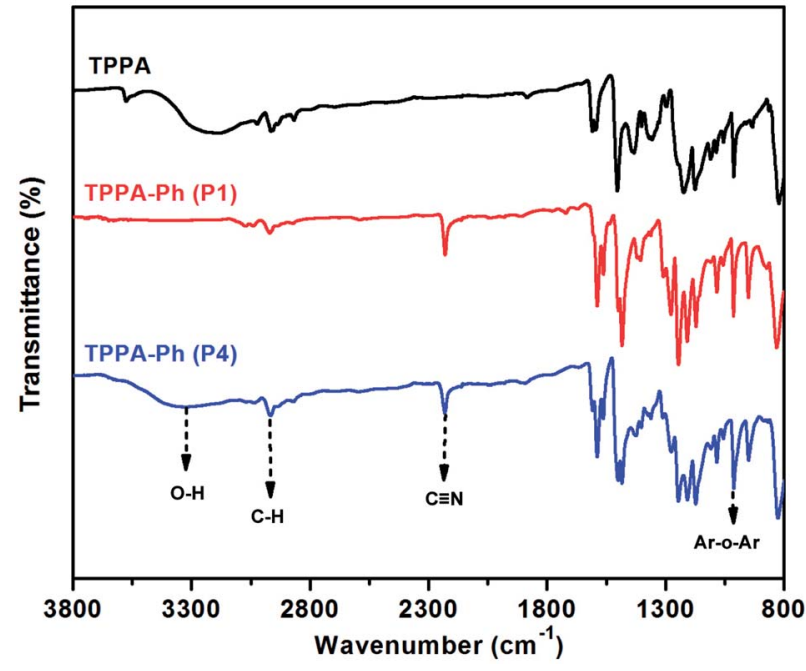

Fig. 2 FT-IR (ATR) spectra of TPPA and TPPA-Ph precursors with different compositions.

investigated using TGA analysis over the temperature range 30$800{ }^{\circ} \mathrm{C}$ at a heating rate of $10{ }^{\circ} \mathrm{C} \mathrm{min}^{-1}$ under a nitrogen atmosphere. The data, including the temperature at $5 \%$ mass loss $\left(T_{\mathrm{d} 5 \%}\right)$ and the char yields at $800{ }^{\circ} \mathrm{C}$, are summarized in Table 2. As shown in Fig. 4, the self-cured TPPA-Ph (P1) resin had the highest $5 \%$ weight loss temperature $\left(T_{\mathrm{d} 5 \%}\right)$ of $506.1^{\circ} \mathrm{C}$, exhibiting a char retention of $81.2 \%$ after heating to $800{ }^{\circ} \mathrm{C}$ under a nitrogen atmosphere. Cured TPPA-Ph resins with different distributions had distinct thermal stabilities, with a decrease in phenolic hydroxyl content in TPPA-Ph precursors

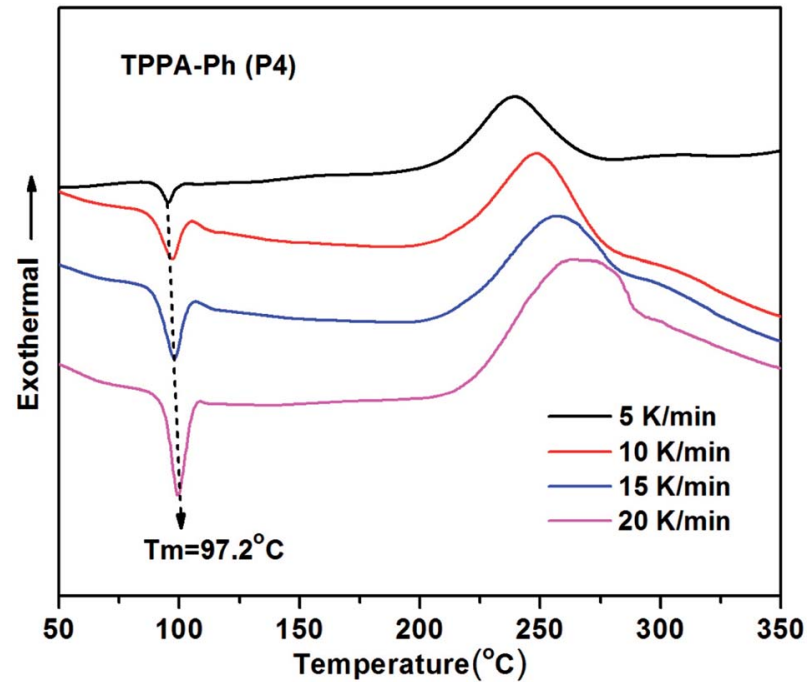

Fig. 3 DSC curing kinetics curves for self-curing TPPA-Ph (P4) under a nitrogen atmosphere at various heating rates.

Table 2 Thermal properties of self-cured TPPA-Ph resins after curing at $400{ }^{\circ} \mathrm{C}$

\begin{tabular}{llll}
\hline TPPA-Ph & $\begin{array}{l}\text { Molar ratio of } \\
\text { residual OH/CN }\end{array}$ & $T_{\mathrm{d} 5 \%\left({ }^{\circ} \mathrm{C}\right)}$ & $\begin{array}{l}\text { Char yield at } \\
800{ }^{\circ} \mathrm{C}(\%)\end{array}$ \\
\hline P1 & 0.03 & 506.1 & 81.2 \\
P2 & 0.22 & 505.7 & 76.8 \\
P3 & 0.35 & 500.3 & 78.1 \\
P4 & 0.55 & 490.6 & 78.5
\end{tabular}


resulting in an increase in $T_{\mathrm{d} 5 \%}$ (see Table 2). Although the presence of residual phenolic hydroxyl groups in TPPA-Ph precursors can promote resin curing, a higher molar ratio of residual phenolic hydroxyl groups to cyano groups will reduce the crosslinking density and thermal stability of the phthalonitrile resin. This suggested that the curing rate and thermal properties of self-cured TPPA-Ph resins can be easily controlled by adjusting the phenolic hydroxyl content in the TPPA-Ph precursors.

The rheological properties of TPPA-Ph (P2) without an additional curing reagent were also investigated. As shown in Fig. 5, the TPPA-Ph (P2) precursor showed a rapid decrease in viscosity at around $100{ }^{\circ} \mathrm{C}$. When the temperature was raised to $140{ }^{\circ} \mathrm{C}$, the viscosity dropped to $50 \mathrm{~Pa} \mathrm{~S}$, which is considered in the machinable range. The viscosity was stable in the range 1$0.1 \mathrm{~Pa} \mathrm{~S}$ with increasing temperature, while the minimum viscosity was $1.615 \mathrm{~Pa} \mathrm{~S}$, which confirmed that the melt of TPPA$\mathrm{Ph}$ resin has satisfactory fluidity. The viscosity levels increased gradually above $256{ }^{\circ} \mathrm{C}$, meaning that the processing temperature window was $116^{\circ} \mathrm{C}$. Therefore, TPPA-Ph resins have a wide processing window and excellent processing fluidity.

\section{Performance of TPPA-Ph system-cured DPPH resins}

TPPA-Ph precursor systems contain phenolic hydroxyl groups and cyano groups that can also be used as curing agents and flexibilizers to optimize the processing performance of phthalonitrile resins. Herein, resorcinol-based phthalonitrile resin precursor DPPH (synthetic route and ${ }^{1} \mathrm{H}$ NMR spectrum shown in Scheme $1 \mathrm{~S}$ and Fig. 5S, $\uparrow$ respectively) was mixed with TPPA$\mathrm{Ph}(\mathrm{P} 4)$ in different mass ratios of $10 \%, 20 \%, 25 \%$, and $30 \%$ and cured at $200{ }^{\circ} \mathrm{C}$ for $2 \mathrm{~h}, 250{ }^{\circ} \mathrm{C}$ for $2 \mathrm{~h}, 300{ }^{\circ} \mathrm{C}$ for $2 \mathrm{~h}, 350{ }^{\circ} \mathrm{C}$ for $2 \mathrm{~h}, 375{ }^{\circ} \mathrm{C}$ for $2 \mathrm{~h}$, and $400{ }^{\circ} \mathrm{C}$ for $2 \mathrm{~h}$ to afford a black green solid. As shown in Fig. 6, after thermal curing, the absorption peak at around $2229 \mathrm{~cm}^{-1}$, attributed to vibration of the cyano group, was diminished. Furthermore, new peaks appeared at around $1010 \mathrm{~cm}^{-1}, 1355 \mathrm{~cm}^{-1}, 1519 \mathrm{~cm}^{-1}$, and $1713 \mathrm{~cm}^{-1}$,

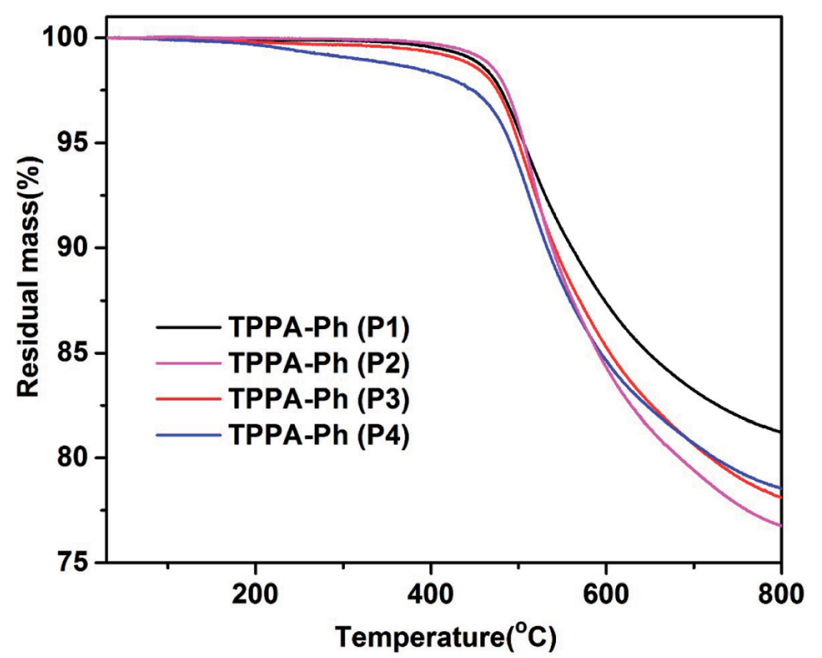

Fig. 4 TGA traces of self-cured resins with different TPPA-Ph contents under a nitrogen atmosphere.

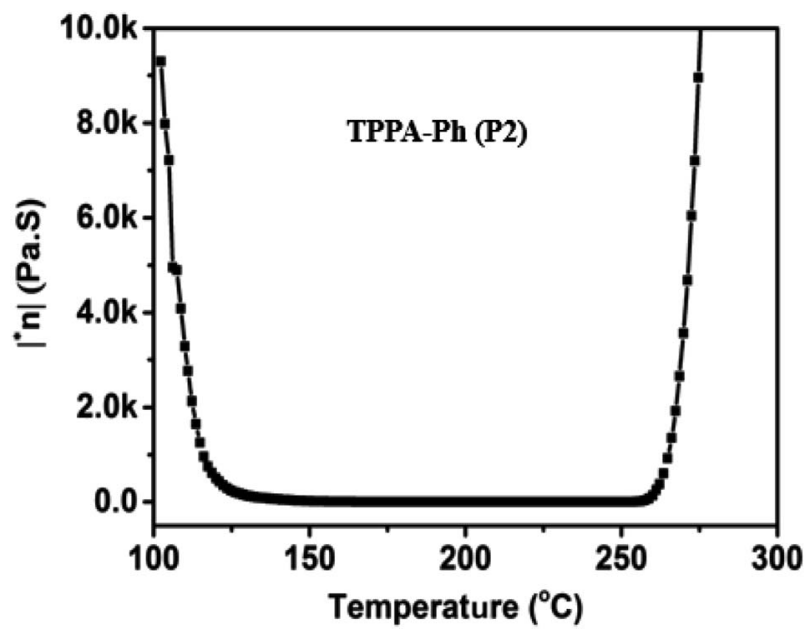

Fig. 5 Complex viscosity $\left(\eta^{*}\right)$ as a function of temperature for TPPA$\mathrm{Ph}(\mathrm{P} 2)$.

which evidenced the formation of phthalocyanine, s-triazine and isoindoline structures, respectively. This suggested that the presence of residual phenolic hydroxyl groups in the TPPA$\mathrm{Ph}$ precursor successfully promoted the curing of resorcinolbased phthalonitrile resin.

The thermal and thermo-oxidative properties of the DPPH resins cured with TPPA-Ph (P4) were evaluated using TGA over the temperature range $30-800{ }^{\circ} \mathrm{C}$ at heating rates of $10{ }^{\circ} \mathrm{C} \min ^{-1}$, as shown in Fig. 7 , and the key data are shown in Table 3. In general, the DPPH resins cured with various contents of TPPA-Ph (P4) possessed excellent thermotolerance, with the temperatures corresponding to $5 \%$ weight loss all exceeding $528{ }^{\circ} \mathrm{C}$ under either nitrogen or air atmospheres, while char yields were all above $76 \%$ when heated to $800{ }^{\circ} \mathrm{C}$ under a nitrogen atmosphere. Although the TPPA-Ph (P4) content was $10 \%$, the $5 \%$ and $10 \%$ weight loss temperatures were the

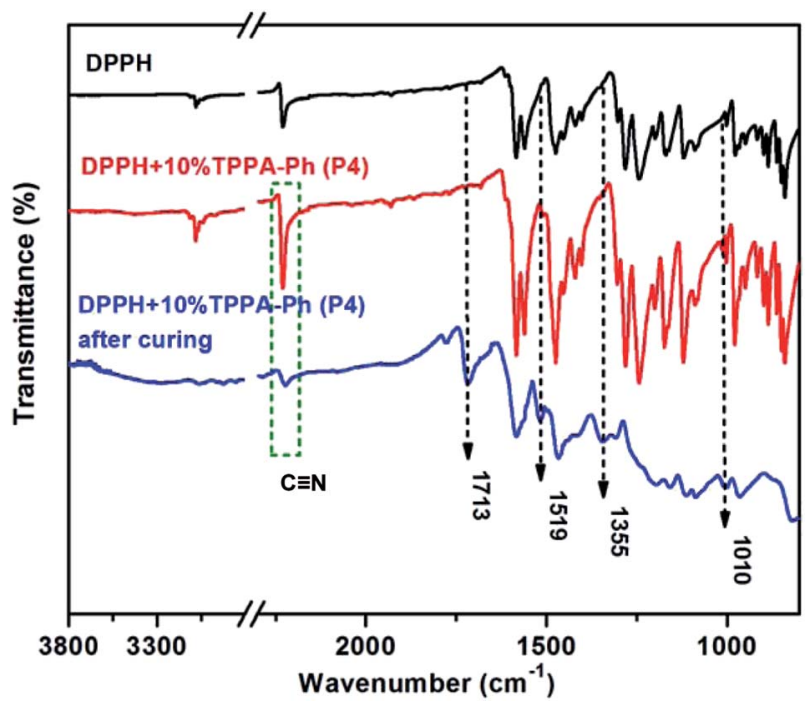

Fig. 6 FT-IR(ATR) spectra of DPPH and DPPH with 10\% TPPA-Ph content before and after curing. 

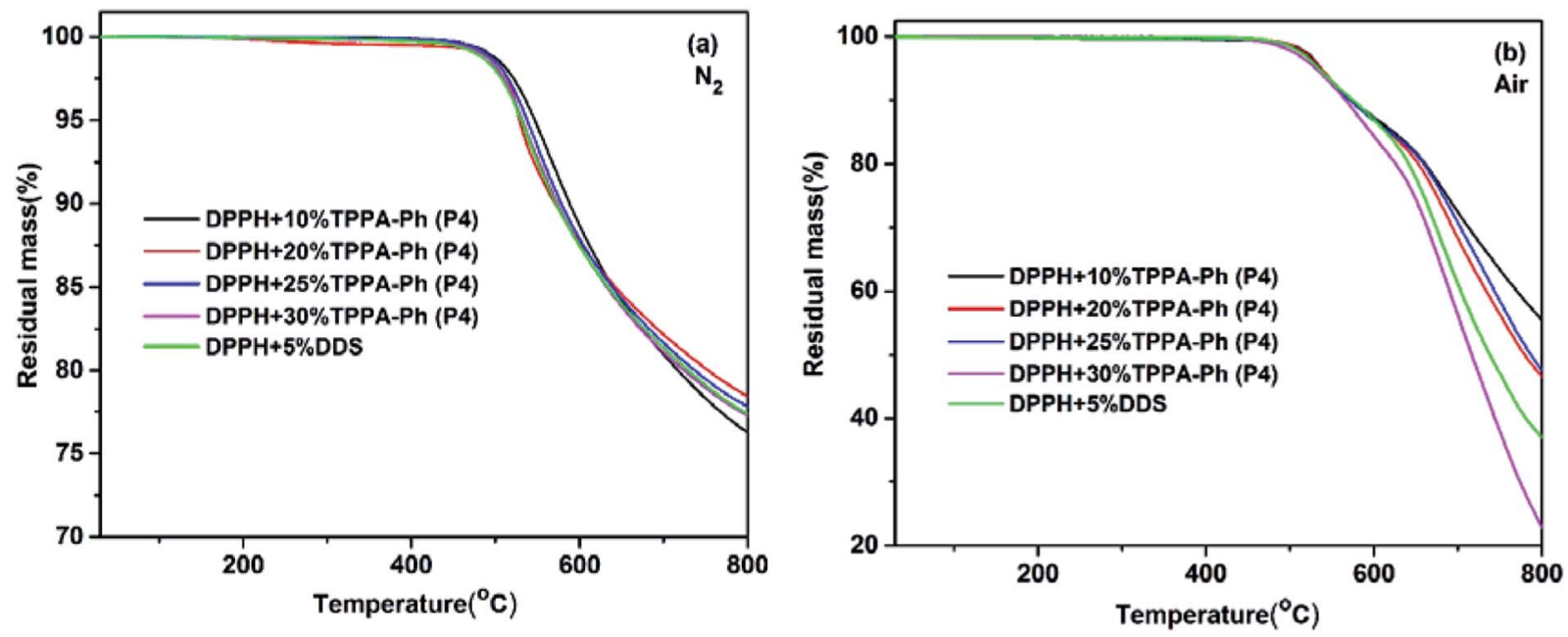

Fig. 7 TGA traces of DPPH resins cured with DDS and different TPPA-Ph contents under (a) nitrogen and (b) air atmospheres.

Table 3 Thermal properties of DPPH resins cured with DDS and different TPPA-Ph (P4) contents

\begin{tabular}{llllll}
\hline & \multicolumn{3}{l}{ In $\mathrm{N}_{2}$} & & \multicolumn{2}{l}{ In air } \\
\cline { 2 - 3 } \cline { 5 - 6 } Curing agent & $\begin{array}{l}T_{\mathrm{d} 5 \%} \\
\left({ }^{\circ} \mathrm{C}\right)\end{array}$ & $\begin{array}{l}\text { Char yield at } \\
800{ }^{\circ} \mathrm{C}(\%)\end{array}$ & & $\begin{array}{l}T_{\mathrm{d} 5 \%} \\
\left({ }^{\circ} \mathrm{C}\right)\end{array}$ & $\begin{array}{l}\text { Char yield at } \\
800{ }^{\circ} \mathrm{C}(\%)\end{array}$ \\
\hline 5\%DDS & 530.9 & 77.4 & 534.4 & 45.3 \\
10\%TPPA-Ph & 547.0 & 76.3 & 537.3 & 55.5 \\
20\%TPPA-Ph & 528.8 & 78.4 & 537.2 & 46.6 \\
25\%TPPA-Ph & 538.0 & 77.8 & 534.6 & 47.5 \\
30\%TPPA-Ph & 532.3 & 77.3 & 531.2 & 22.8
\end{tabular}

highest in any atmosphere, and were also higher than those of DPPH cured with 5\% DDS as reference.

The influence of long-term oxidative aging at high temperature under an air atmosphere is also an important consideration for phthalonitrile resin use. The conditions were simulated by subjecting DPPH resins cured with TPPA-Ph (P4) to stepwise heating at a series of temperatures between 200 and $375{ }^{\circ} \mathrm{C}$ at $8 \mathrm{~h}$ intervals under air. Fig. 8 shows the percentage residual mass after weight loss at various temperatures. A cumulative weight loss of 1.68-3.84\% was observed for a TPPA$\mathrm{Ph}(\mathrm{P} 4)$ content range of $10-30 \%$. This performance was similar to that of the resin with DDS as curing agent. Based on the above results, the DPPH resins cured with TPPA-Ph (P4) showed outstanding thermal stability and thermo-oxidative stability.

Rheological behavior plays an important role in confirming the processability of resins. The processing window and time required for curing were further investigated by examining the complex viscosity $\left(\eta^{*}\right)$ of DPPH resins cured with TPPA-Ph (P4) as a function of temperature from $160{ }^{\circ} \mathrm{C}$ to $370{ }^{\circ} \mathrm{C}$. As shown in Fig. 9a, the viscosity of the resins was influenced by the TPPA-Ph (P4) content, with TPPA-Ph (P4) contents of 10\%, 20\%, 25\%, and $30 \%$ showing rapid complex viscosity increases at $330{ }^{\circ} \mathrm{C}$, $290{ }^{\circ} \mathrm{C}, 270^{\circ} \mathrm{C}$, and $260{ }^{\circ} \mathrm{C}$, respectively. At temperatures above $175^{\circ} \mathrm{C}$, a minimum melt complex viscosity of around $0.2 \mathrm{~Pa} \mathrm{~S}$ was observed for all DPPH resins. The low melt viscosity of the resins at higher temperatures was evidence of their superb melt fluidity and stability. However, for the DDS curing system, the lowest viscosity was around $0.08 \mathrm{~Pa} \mathrm{~S}$ at $191^{\circ} \mathrm{C}$, and the curing window was narrower than that of the TPPA-Ph system. In Fig. 9b, complex viscosity measurements as a function of time for DPPH resins with different TPPA-Ph (P4) contents were performed at $250{ }^{\circ} \mathrm{C}$, respectively. The processing window and time of the DPPH resins could be adjusted by the TPPA-Ph (P4) content. Based on the above results, the DPPH resins with TPPA$\mathrm{Ph}$ (P4) showed good processability with low complex viscosity and a wide processing window at moderate temperatures.

The dynamic mechanical properties are another fundamental feature that determines the upper limit of the application temperature in developing high-performance phthalonitrile resin. The dynamic mechanical properties of DPPH resins cured with TPPA-Ph (P4) were investigated to evaluate changes in the resin modulus as a function of

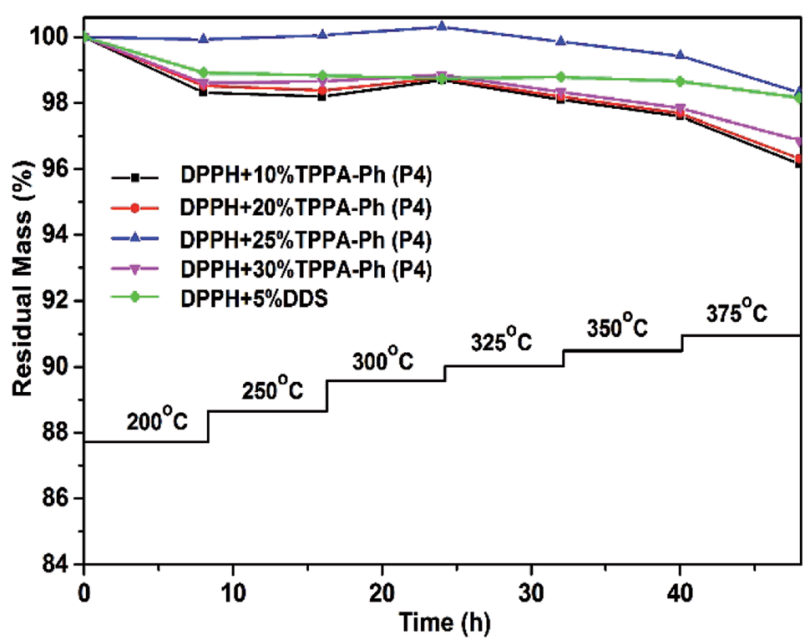

Fig. 8 Long-term oxidative aging plots of DPPH phthalonitrile resins cured with DDS and different TPPA-Ph (P4) contents. 

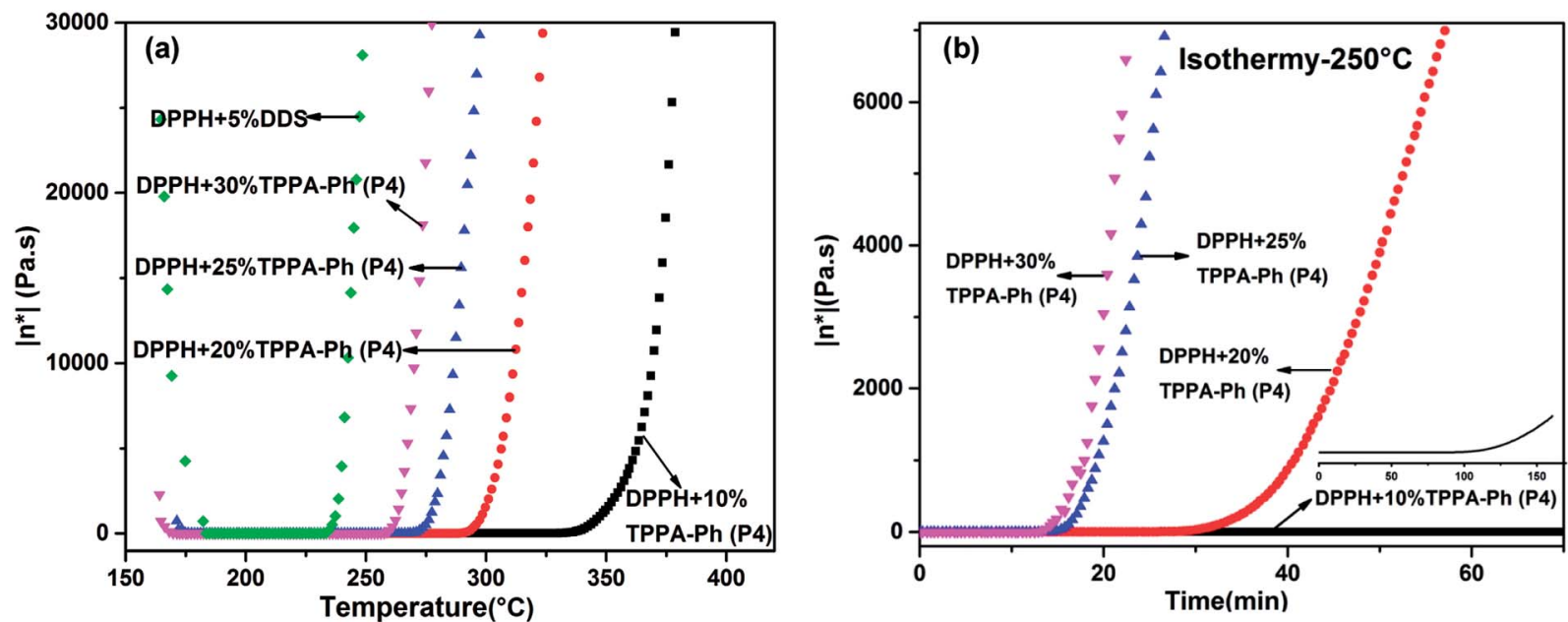

Fig. 9 Complex viscosity $\left(\eta^{*}\right)$ as a function of (a) temperature and (b) time for cured DPPH phthalonitrile resins with DDS and different TPPA-Ph (P4) contents.
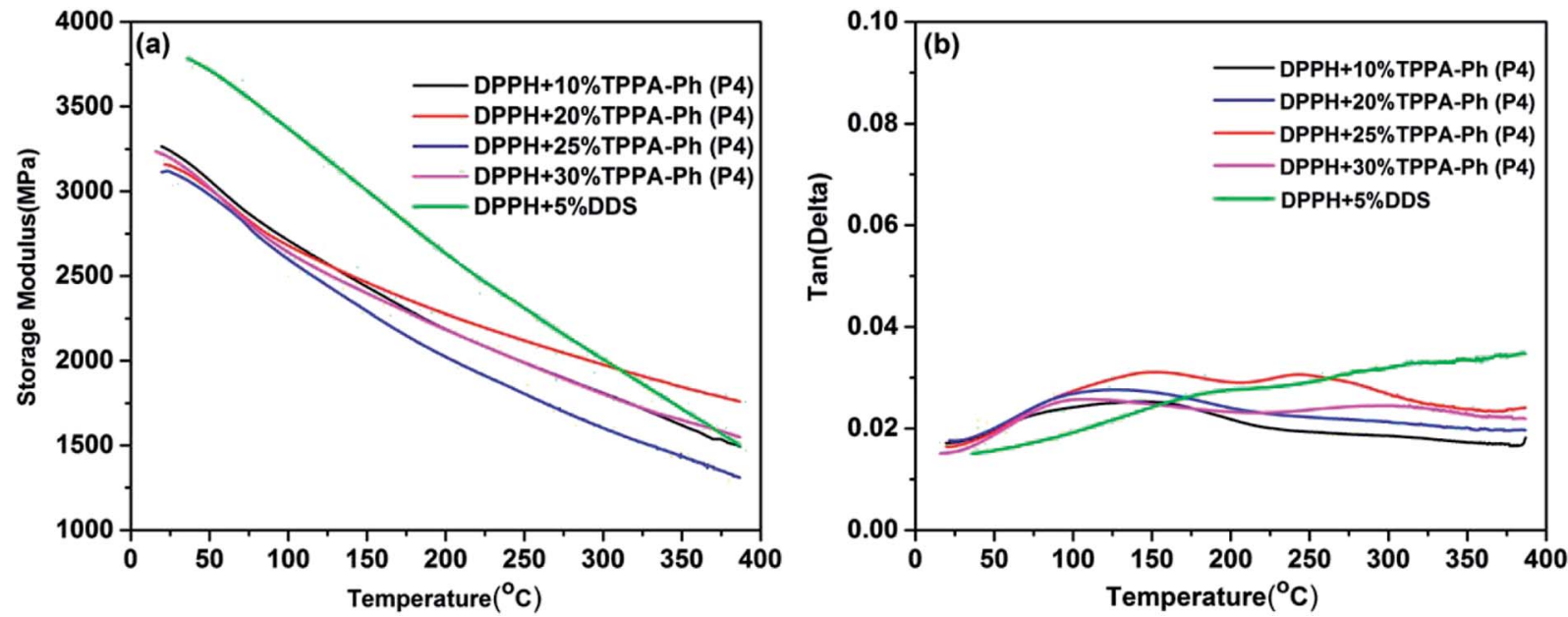

Fig. 10 Dynamic mechanical properties of DPPH resins cured with DDS and different contents of TPPA-Ph (P4): (a) storage modulus vs. temperature; (b) $\tan \delta$ vs. temperature.

temperature and to determine the glass transition temperature of DPPH resins. The dynamic mechanical behavior of resins, including storage modulus $\left(G^{\prime}\right)$ and damping factor $(\tan \delta)$ at a fixed frequency of $1 \mathrm{~Hz}$ in single cantilever mode, are shown in Fig. 10. All cured resins exhibited a high storage modulus of more than $3.0 \mathrm{GPa}$ at $50{ }^{\circ} \mathrm{C}$, which decreased as the temperature gradually increased. This high modulus should be due to heteroaromatic structures and the high degree of crosslinking. Furthermore, the declining modulus with increasing temperature was attributed to stress relaxation of the cured resins. The $\tan d v s$. temperature curves for DPPH cured resins are shown in Fig. 10b, showing that the $\tan \delta$ values were below 0.03 when heated to $380{ }^{\circ} \mathrm{C}$ with no obvious relaxation transition, suggesting that the glass transition temperature of DPPH resins were all above $380{ }^{\circ} \mathrm{C}$ within the testing range. Some small transitions were ascribed to secondary relaxation of the phthalonitrile structures. As determined above, DPPH resins cured with various TPPA-Ph (P4) contents possessed excellent thermal stability. For DPPH cured with DDS, no $T_{\mathrm{g}}$ was detected within the tested temperature range and the storage modulus was much higher than that using the TPPA-Ph system due to the rigid structure of DDS.

Another important factor in the use of resins under real conditions is water absorption caused by the cyano and phenolic hydroxyl groups. The water absorption was investigated by immersing the DPPH resins cured with TPPA-Ph (P4) in boiling deionized water for $100 \mathrm{~h}$. Data, including the weight increase at $100 \mathrm{~h}$, are summarized in Fig. 11. However, the water absorption of all resins with different TPPA-Ph (P4) contents was less than $4 \%$, which was lower than that of the DDS curing system, guaranteeing the effective use of DPPH resin in practical production. 


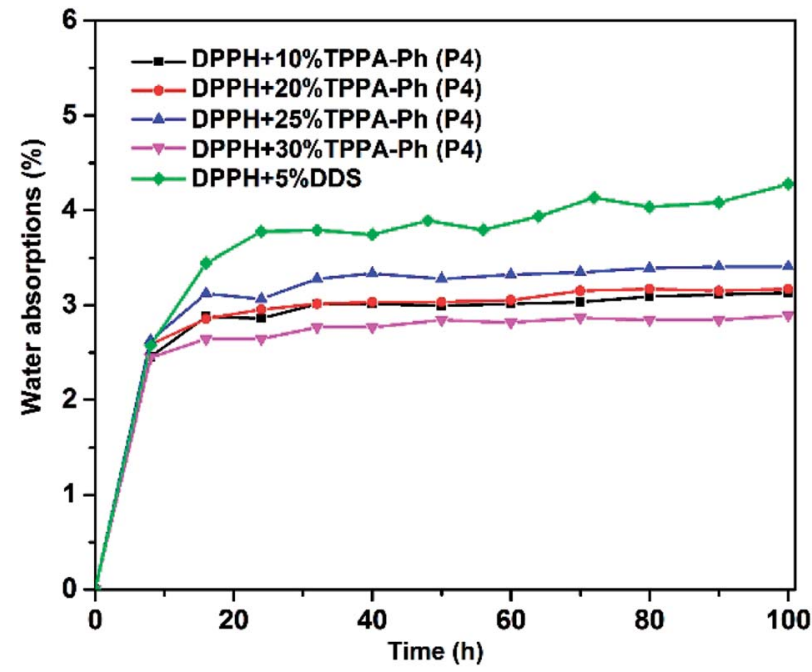

Fig. 11 Moisture absorption curves for DPPH phthalonitrile resins cured with DDS and different TPPA-Ph (P4) contents.

\section{Conclusions}

In this research, a novel multifunctional self-curing triphenol Abased phthalonitrile resin precursor was successfully synthesized from TPPA and NPh. The reaction conditions afforded a mixture of five TPPA-Ph compounds containing different amounts of underivatized phenolic hydroxyl groups, which act as curing centers to facilitate curing of the TPPA-based phthalonitrile resin in the absence of an additional curing reagent. The melting point of self-curing TPPA-Ph precursors was less than $100{ }^{\circ} \mathrm{C}$, and the thermal properties and processing window of self-cured TPPA-Ph resins was regulated by adjusting the phenolic hydroxyl content in TPPA-Ph precursors. Furthermore, TPPA-Ph precursor systems contained phenolic hydroxyl groups and flexible structures that can act as curing agents and flexibilizers to optimize other phthalonitrile resins. For example, resorcinol-based phthalonitrile resin (DPPH) cured with various TPPA-Ph (P4) contents possessed excellent thermal stability and thermo-oxidative stability, and a wide processing window. Therefore, this study provides a strategy for constructing a multifunctional phthalonitrile resin precursor that can improve the curing process without sacrificing other properties, such as thermal stability and thermo-oxidative stability.

\section{Conflicts of interest}

There are no conflicts to declare.

\section{Acknowledgements}

This work was supported by the National Nature Science Foundation of China (Nos. 51673033, U1663226 and 51873027) and the Fundamental Research Funds for the Central Universities (DUT17LK39).

\section{References}

1 A. Rawal, P. V. Kameswara, S. Russell and A. Jeganathan, J. Appl. Polym. Sci., 2010, 116, 2668-2673.

2 S. B. Sastri, J. P. Armistead and T. M. Keller, Polym. Compos., 1996, 17, 816-822.

3 S. B. Sastri, J. P. Armistead and T. M. Keller, Polym. Compos., 1997, 18, 48-54.

4 F. Zuo and X. B. Liu, J. Appl. Polym. Sci., 2010, 117, 14691475.

5 T. M. Keller, SAMPE Symp. Proc., 1986, 31, 528-532.

6 T. M. Keller, CHEMTECH, 1988, 18, 635-639.

7 J. F. Giuliani and T. M. Keller, Sens. Mater., 1989, 1, 22-27.

8 A. Badshah, M. R. Kessler, Z. Heng, J. H. Zaidi, S. Hameed and A. Hasan, Polym. Chem., 2013, 4, 3617-3622.

9 W. H. Zhu and G. S. Wu, J. Phys. Chem. A, 2001, 105, 95689574.

10 T. M. Keller and D. D. Dominguez, Polymer, 2007, 48, 91-97. 11 S. B. Sastri and T. M. Keller, J. Polym. Sci., Part A: Polym. Chem., 1998, 36, 1885-1890.

12 T. M. Keller, Polym. Prepr., 1992, 33, 422-423.

13 T. M. Keller and T. R. Price, J. Macromol. Sci., Chem., 1982, 18, 931-937.

14 P. J. Burchill, J. Polym. Sci., Part A: Polym. Chem., 1994, 32, 18.

15 T. R. Walton, J. R. Griffith and J. G. O'Rear, J. Adhes. Sci. Technol., 1975, 9, 665-676.

16 L. Y. Sun and Z. T. Huang, Chin. J. Polym. Sci., 1990, 8, 177182.

17 S. B. Sastri and T. M. Keller, J. Polym. Sci., Part A: Polym. Chem., 1998, 36, 1885-1890.

18 T. M. Keller, Chem. Mater., 1994, 6, 302-305.

19 T. M. Keller, Polymer, 1993, 34, 952-955.

20 T. M. Keller and T. R. Price, Polym. Commun., 1984, 25, 4244.

21 T. M. Keller and D. D. Dominguez, Polymer, 2005, 46, 46144618.

22 X. Fu, G. P. Yu, C. Liu, J. Y. Wang, C. Y. Pan and X. G. Jian, High Perform. Polym., 2014, 26, 540-549.

23 X. Y. Yu, K. Naito, C. Kang, X. W. Qu and Q. X. Zhang, Macromol. Chem. Phys., 2013, 214, 361-369.

24 T. M. Robert, D. Augustine, S. Chandran, D. Mathew and C. P. R. Nair, RSC Adv., 2015, 5, 1198-1204.

25 H. T. Sheng, X. G. Peng, H. Guo, X. Y. Yu, K. Naito and X. W. Qu, Thermochim. Acta, 2014, 577, 17-24.

26 M. Laskoski, A. Neal, T. M. Keller, D. D. Dominguez, C. A. Klug and A. P. Saab, J. Polym. Sci., Part A: Polym. Chem., 2014, 52, 1662-1668.

27 G. P. Yu, C. Liu, X. P. Li, J. Y. Wang, X. G. Jian and C. Y. Pan, Polym. Chem., 2012, 3, 1024-1032.

28 Z. Q. Wu, J. H. Han, N. Li, Z. H. Weng, J. Y. Wang and X. G. Jian, Polym. Int., 2017, 66, 876-881.

29 Z. H. Weng, J. Y. Fu, L. S. Zong, C. Liu, J. Y. Wang and X. G. Jian, RSC Adv., 2015, 5, 92055-92060.

30 S. Vyazovkin, Thermochim. Acta, 2000, 355, 155-163. 
31 C. F. Dickinson and G. R. Heal, Thermochim. Acta, 2009, 494, 15-25.

32 S. Vyazovkin, A. K. Burnham, J. M. Criado, L. A. PerezMaqueda, C. Popescu and N. Sbirrazzuoli, Thermochim. Acta, 2011, 520, 1-19.

33 K. Zeng, K. Zhou, S. H. Zhou, H. B. Hong, H. F. Zhou and Y. P. Wang, Eur. Polym. J., 2009, 45, 1328-1335.

34 M. Z. Xu, M. D. Liu, S. H. Dong and X. B. Liu, J. Mater. Sci., 2013, 48, 8108-8116.
35 Z. Lei and H. Xiao, Polymer, 2010, 51, 3814-3820.

36 C. Jubsilp, S. Damrongsakkul, T. Takeichi and S. Rimdusit, Thermochim. Acta, 2006, 447, 131-140.

37 T. Zhang, J. Wang, M. Derradju, N. Ramdani, H. Wang and Z. W. Lin, Thermochim. Acta, 2015, 602, 22-29.

38 M. Z. Xu, Y. S. Luo, Y. X. Lei and X. B. Liu, Polym. Test., 2016, $55,38-43$.

39 G. M. Roudsari, A. K. Mohanty and M. Misra, ACS Sustainable Chem. Eng., 2014, 2, 2111-2116. 